\title{
Failure of Parameter Identification Based on Adaptive Synchronization Techniques
}

\author{
Wei LIN $\ddagger$ and Huan-Fei MA \\ Key Laboratory of Mathematics for Nonlinear Sciences (Fudan University), \\ Ministry of Education, Research Center for Nonlinear Sciences, School of \\ Mathematical Sciences, Fudan University, Shanghai 200433, China.
}

$\ddagger$ To whom correspondence should be addressed:

Dr. Wei LIN,

School of Mathematical Sciences

Fudan University

220 HanDan Rd.

Shanghai 200433

CHINA

Phone: 86-21-5566-4901

Fax: 86-21-6564-2342

E-mail: wlin@fudan.edu.cn 


\begin{abstract}
In the paper, several concrete examples, as well as their numerical simulations, are given to show that parameter identification based on the so-called adaptive synchronization techniques might be failed if those functions with parameters pending for identification in coupled systems are designed to be mutually linearly dependent or approximately linearly dependent on the orbit in the synchronization manifold. This failure might be emergent not only when the synchronized orbit is selected to be some sort of equilibrium or some sort of periodic oscillation, but also when it is taken as some type of chaotic attractor produced by driving system. This result implies that chaotic property of driving signal is not necessary to achievement of parameter identification. The mechanism inducing such a failure, as well as the bounded property of all trajectories generated by coupled systems, is theoretically expatiated. New synchronization techniques are proposed to rigorously realize the complete synchronization and parameter identification in a class of systems where the nonlinearity is not globally Lipschitz. In addition, parameter identification are discussed for systems with time delay.
\end{abstract}

PACS: 05.45.Gg, 05.40.-a, 87.10.+e. 


\section{Introduction}

The most classical phenomenon in reference to synchronization is owing to Huygens' observation about the synchrony of pendulum clocks [1]. Since this historical discovery, synchronization as an omnipresent technical issue has become a focal topic of great importance in many applications. Moreover, the basic concept related to chaos synchronization in coupled chaotic systems was initially introduced by Pecora and Carrol in 1990 [2]. Since their seminal paper, chaos synchronization as an interesting research topic of great potential application has been widely investigated and consequently applied in plenty of fields, ranging from secure communications to pattern recognitions, from complex network dynamics to optimization of nonlinear systems, and even from chemical reaction to brain activity analysis [3]. In particular, a wide varieties of synchronization approaches, including traditional linear or nonlinear feedback coupling, impulse coupling, invariant manifold method, adaptive design coupling techniques, and white-noise-based coupling have been fruitfully proposed [4]-[5] and several types of synchronization, including complete synchronization, generalized synchronization, phase synchronization, and lag synchronization, have been introduced in succession [6]-[9].

Among all the proposed coupling approaches for realization of complete synchronization between coupled chaotic systems with or without time delays, the newly developed adaptive design coupling technique has aroused a great amount of attention from many researchers [10]-18 simply due to the reported success in unknown parameter identification. Their explorations have shown that unknown parameters could be identified in several well-known chaotic systems and even in some neural network models with or without time delays. In particular, consider an $n$-dimensional nonlinear system described by

$$
\dot{\boldsymbol{x}}=\boldsymbol{F}(\boldsymbol{x}, \boldsymbol{p}),
$$

where $\boldsymbol{x}=\left(x_{1}, x_{2}, \ldots, x_{n}\right)^{T} \in \mathbb{R}^{n}, \boldsymbol{F}(x, \boldsymbol{p})=\left(F_{1}(\boldsymbol{x}, \boldsymbol{p}), F_{2}(\boldsymbol{x}, \boldsymbol{p}), \ldots, F_{n}(\boldsymbol{x}, \boldsymbol{p})\right)^{T}$, and

$$
F_{i}(\boldsymbol{x}, \boldsymbol{p})=c_{i}(\boldsymbol{x})+\sum_{j=1}^{m} p_{i j} f_{i j}(\boldsymbol{x}), \quad i=1,2, \ldots, n .
$$

Here, $c_{i}(\boldsymbol{x})$ and $f_{i j}(\boldsymbol{x})$ are, respectively, assumed to be some kind of real valued functions, and $\boldsymbol{p}=\left\{p_{i j}\right\} \in \mathcal{U} \subset \mathbb{R}^{n}$ are $(n \cdot m)$ parameters pending for identification, in which $\mathcal{U}$ 
is some bounded set. Given the bounded driving signal $\boldsymbol{x}(t)$ produced by system (1), its response system is designed through

$$
\begin{aligned}
& \dot{\boldsymbol{y}}=\boldsymbol{F}(\boldsymbol{y}, \boldsymbol{q})+\boldsymbol{\epsilon} \cdot \boldsymbol{e}, \\
& \dot{\epsilon}_{i}=-r_{i} e_{i}^{2}, \quad \dot{q}_{i j}=-\delta_{i j} e_{i} f_{i j}(\boldsymbol{y}), \\
& i=1,2, \ldots, n, j=1,2, \ldots, m,
\end{aligned}
$$

where the feedback coupling $\boldsymbol{\epsilon} \cdot \boldsymbol{e}$ is in the form of $\left(\epsilon_{1} e_{1}, \epsilon_{2} e_{2}, \ldots, \epsilon_{n} e_{n}\right)^{T}, e_{i}=\left(y_{i}-x_{i}\right)$, $\boldsymbol{q}=\left\{q_{i j}\right\}$, and both $r_{i}$ and $\delta_{i j}$ are arbitrarily chosen positive constants.

A question naturally arises: "Is it possible to accurately identify all the $(n \cdot m)$ parameters of the chaotic system provided that the output time series of system (1) are experimentally obtained?" When the response system is designed as (3), the answer to this question, as mentioned above, is reportedly positive. Concretely, the complete synchronization between the driving system (10) and the response system (3) could be always achieved; moreover, the varying parameters $\boldsymbol{q}$ in (3), initiating from arbitrary values, will be asymptotically convergent to the correct values of the parameters $\boldsymbol{p}$ in (1) as time tends towards positive infinity. Seemingly, their theoretical arguments are based on a delicate design for Lyapunov function, on the well-known Lyapunov Stability theorem, and even on the LaSalle invariance principle.

As a matter of fact, those signals produced by these driving-and-response systems could be completely synchronized; nevertheless, the parameter identification might be failed if those terms with parameters pending for identification are designed to be mutually linearly dependent or approximately linearly dependent on the synchronized orbit. In the paper, not only concrete examples with their numerical simulations will be provided to illustrate such a failure of parameter identification, but also the mechanism inducing this failure will be anatomized. Also, the performed analysis will show that chaotic property of synchronized orbit in synchronization manifold (or say, chaotic property of positive limit set of driving signal) is not always necessary to achievement of parameter identification.

The rest of the paper is organized as follows. In Section 2, three concrete examples, as well as their numerical simulations, are consecutively given to illustrate the possible occurrence of parameter identification failure. The synchronized orbits in those examples 
are, respectively, selected to be some sort of equilibrium, periodic oscillation, and chaotic attractor. The mechanism inducing this failure, as well as the the bounded property of all trajectories generated by the coupled systems (10) and (3), is theoretically expatiated in Section 3. Furthermore, in Section 4, new synchronization techniques are further proposed to realize complete synchronization and parameter identification in a class of polynomial systems where the nonlinearity is not globally Lipschitz. In Section 5, parameter identification are discussed for systems with time delay. Finally, the paper is closed with some concluded remarks.

\section{Examples Showing Failure of Parameter Identification}

In this section, three groups of driving-and-response systems are concretely presented to illustrate the possible occurrence of failed parameter identification.

First, consider the Lorenz system:

$$
\begin{aligned}
& \dot{x}_{1}=p_{1}\left(x_{2}-x_{1}\right), \\
& \dot{x}_{2}=p_{2} x_{1}-x_{1} x_{3}-x_{2}, \\
& \dot{x}_{3}=x_{1} x_{2}-p_{3} x_{3}
\end{aligned}
$$

as a driving system. And the corresponding response system becomes:

$$
\begin{aligned}
& \dot{y}_{1}=q_{1}\left(y_{2}-y_{1}\right)+\epsilon_{1}\left(y_{1}-x_{1}\right), \\
& \dot{y}_{2}=q_{2} y_{1}-y_{1} y_{3}-y_{2}+\epsilon_{2}\left(y_{2}-x_{2}\right), \\
& \dot{y}_{3}=y_{1} y_{2}-q_{3} y_{3}+\epsilon_{3}\left(y_{3}-x_{3}\right)
\end{aligned}
$$

where the updating laws of $\boldsymbol{q}=\left(q_{1}, q_{2}, q_{3}\right)$ and $\boldsymbol{\epsilon}=\left(\epsilon_{1}, \epsilon_{2}, \epsilon_{3}\right)$ are, respectively, taken as: $\dot{q}_{1}=-\delta_{1}\left(y_{1}-x_{1}\right)\left(y_{2}-y_{1}\right), \dot{q}_{2}=-\delta_{2}\left(y_{2}-x_{2}\right) y_{2}, \dot{q}_{3}=-\delta_{3}\left(y_{3}-x_{3}\right)\left(-y_{3}\right)$, and $\dot{\epsilon}_{1}=-r_{1}\left(y_{1}-x_{1}\right)^{2}, \dot{\epsilon}_{2}=-r_{2}\left(y_{2}-x_{2}\right)^{2}, \dot{\epsilon}_{3}=-r_{3}\left(y_{3}-x_{3}\right)^{2}$.

Particularly, when the parameters are taken as $p_{1}=35, p_{2}=\frac{8}{3}$, and $p_{3}=28$, the complete synchronization between systems (4) and (5) could be achieved with time evolution, which is numerically shown by Fig.1(a). This group of parameters, which are different from the classical parameters inducing strange attractor of the Lorenz system, simply 
make the synchronized orbit generated by system (4) become an asymptotically stable equilibrium $E$, as is shown by Fig.1(b). If the reported analytical results are completely correct, it could be expected that the varying parameters $\boldsymbol{q}$ will be eventually convergent to the accurate values of the parameters $\boldsymbol{p}=\left(p_{1}, p_{2}, p_{3}\right)$. However, this is not the case. As depicted in Fig.2, although the numerical simulation is consistent with the expectation for the parameters $q_{2,3}$, it is beyond the expectation for the parameters $q_{1}$. Concretely, the value of $q_{1}$ does not approach but always keeps a distant from the accurate value of $p_{1}$ with time evolution, which means failed parameter identification does occur for $q_{1}$. Intuitively, there must exist some mechanism inducing such differences between $q_{1}$ and $q_{2,3}$ when adaptive synchronization techniques are taken into account.

Secondly, construct a driving system based on the Chen's system through:

$$
\begin{aligned}
& \dot{x}_{1}=30\left(x_{2}-x_{1}\right)+\mathcal{Q}\left(x_{1}, x_{2}, p_{1}, p_{2}\right), \\
& \dot{x}_{2}=(28-30) x_{1}-x_{1} x_{3}+28 x_{2}, \\
& \dot{x}_{3}=x_{1} x_{2}-3 x_{3} .
\end{aligned}
$$

where the additional term

$$
\begin{aligned}
\mathcal{Q}\left(x_{1}, x_{2}, p_{1}, p_{2}\right)= & 0.1 \times\left\{p_{1} \frac{\left(x_{1} \cos (0.9026)+x_{2} \sin (0.9026)\right)^{2}}{23.44^{2}}\right. \\
& \left.-p_{2}\left[\frac{\left(-x_{1} \sin (0.9026)+x_{2} \cos (0.9026)\right)^{2}}{7.19^{2}}-1\right]\right\},
\end{aligned}
$$

and both $p_{1}$ and $p_{2}$ are parameters expected to be identified. As a matter of fact, without the term $\mathcal{Q}$, system (6) becomes the original Chen's system admitting an attractive periodic orbit. As displayed in Fig.3, the projection of this attractive periodic orbit into the $x_{1}-x_{2}$ plane is approximately looked upon as an ellipse. Thus, when $p_{1}=1$ and $p_{2}=-1$, the term $\mathcal{Q}$ actually is an approximate formula of this projection in the $x_{1}-x_{2}$ plane.

Given a driving signal $\left(x_{1}, x_{2}, x_{3}\right)^{T}$ generated by system (주), the corresponding response system is designed to be in the form of

$$
\begin{aligned}
& \dot{y}_{1}=30\left(y_{2}-y_{1}\right)+\mathcal{Q}\left(y_{1}, y_{2}, q_{1}, q_{2}\right)+\epsilon_{1}\left(y_{1}-x_{1}\right), \\
& \dot{y}_{2}=(28-30) y_{1}-y_{1} y_{3}+28 y_{2}+\epsilon_{2}\left(y_{2}-x_{2}\right), \\
& \dot{y}_{3}=y_{1} y_{2}-3 y_{3}+\epsilon_{3}\left(y_{3}-x_{3}\right) .
\end{aligned}
$$


in which, according to (3), the updating laws of the two varying parameters are taken as:

$$
\begin{aligned}
& \dot{q}_{1}=-\delta_{1}\left(y_{1}-x_{1}\right)\left[\frac{\left(y_{1} \cos (0.9026)+y_{2} \sin (0.9026)\right)^{2}}{23.44^{2}}\right], \\
& \dot{q}_{2}=-\delta_{2}\left(y_{1}-x_{1}\right)\left[-\frac{\left(-y_{1} \sin (0.9026)+y_{2} \cos (0.9026)\right)^{2}}{7.19^{2}}+1\right],
\end{aligned}
$$

and the adaptive techniques of coupling strengths are, respectively, chosen as: $\dot{\epsilon}_{1}=$ $-r_{1}\left(y_{1}-x_{1}\right)^{2}, \dot{\epsilon}_{2}=-r_{2}\left(y_{2}-x_{2}\right)^{2}$, and $\dot{\epsilon}_{3}=-r_{3}\left(y_{3}-x_{3}\right)^{2}$.

Contrary to the expectation, both $q_{1}$ and $q_{2}$, starting from almost every initial values, fail to approach the real values of the parameters $p_{1}=1$ and $p_{2}=-1$, as shown in Fig.4. This example, as well as the first example, shows that failure does occur for parameter identification based on the adaptive synchronization technique when the synchronized orbit is particularly selected to be some type of steady dynamics, such as asymptotically stable equilibrium and attractive periodic orbit.

Instead of the above-mentioned steady synchronized orbit, the existing numerical results [10]-[18] show that parameter identification could be always achieved when those synchronized orbits in the synchronization manifold are designed to be some type of chaotic attractor in advance. Then, a question arises: "Is chaotic property of synchronized orbit in synchronization manifold necessary to achievement of parameter identification based on the adaptive techniques?"

To find out an answer to this question, consider a 4-dimensional model developed from the original chaotic Lorenz system as a driving system:

$$
\begin{aligned}
& \dot{x}_{1}=p_{1}\left(x_{2}-x_{1}\right), \\
& \dot{x}_{2}=p_{2} x_{1}-x_{1} x_{3}-x_{2}, \\
& \dot{x}_{3}=x_{1} x_{2}-p_{3} x_{3}+p_{4} x_{3}\left(1+x_{4}^{3}\right), \\
& \dot{x}_{4}=a x_{4}+b\left(x_{1}-x_{3}\right),
\end{aligned}
$$

where $p_{1}=10, p_{2}=28$, and $p_{3}=\frac{8}{3}$ are three classical parameters for the original Lorenz system to generate chaotic attractor, and $a=-100, b=0.1, p_{4}=1$. Given these parameters, the orbit produced by system (8) in the synchronization manifold still exhibits chaotic property in the phase plane, which is displayed by Fig.5(a)-(b). This 
chaotic property is further verified by calculating the largest Lyapunov exponent of the system $\left(\lambda_{1} \approx 0.54274>0\right)$, as is shown by Fig.5(c).

Provided with the driving signal produced by system (8), the complete synchronization between systems (8) and its response system could be numerically achieved as long as the response system is designed through:

$$
\begin{aligned}
& \dot{y}_{1}=q_{1}\left(y_{2}-y_{1}\right)+\epsilon_{1}\left(y_{1}-x_{1}\right), \\
& \dot{y}_{2}=q_{2} y_{1}-y_{1} y_{3}-y_{2}+\epsilon_{2}\left(y_{2}-x_{2}\right), \\
& \dot{y}_{3}=y_{1} y_{2}-q_{3} y_{3}+q_{4} y_{3}\left(1+y_{4}^{3}\right)+\epsilon_{3}\left(y_{3}-x_{3}\right), \\
& \dot{y}_{4}=a y_{4}+b\left(y_{1}-y_{3}\right)+\epsilon_{4}\left(y_{4}-x_{4}\right),
\end{aligned}
$$

in which the updating laws of the parameters are taken as $\dot{q}_{1}=-\delta_{1}\left(y_{1}-x_{1}\right)\left(y_{2}-y_{1}\right), \dot{q}_{2}=$ $-\delta_{2}\left(y_{2}-x_{2}\right) y_{1}, \dot{q}_{3}=-\delta_{3}\left(y_{3}-x_{3}\right)\left(-y_{3}\right), \dot{q}_{4}=-\delta_{4}\left(y_{3}-x_{3}\right)\left[y_{3}\left(1+y_{4}^{3}\right)\right]$, and the adaptive coupling strengths are taken as $\dot{\epsilon}_{1}=-r_{1}\left(y_{1}-x_{1}\right)^{2}, \dot{\epsilon}_{2}=-r_{2}\left(y_{2}-x_{2}\right)^{2}, \dot{\epsilon}_{3}=-r_{3}\left(y_{3}-x_{3}\right)^{2}$, $\dot{\epsilon}_{4}=-r_{4}\left(y_{4}-x_{4}\right)^{2}$. In spite of the success in complete synchronization and in parameter identification for $q_{1,2}$, it is impossible to utilize $q_{3,4}$, initiating from almost every points, to identify the accurate values of the parameters $p_{3,4}$ in system (8) . All these are shown in Fig.6. Clearly, this example implies that the answer to the above-posed question is negative.

Remark 2.1 The fourth-order Runge-Kutta scheme is used to solve all the ordinary differential equations in our numerical simulations.

\section{The Mechanism Inducing the Failure}

On the one hand, three concrete examples in the last section show that some parameter identification might be failed no matter what kind of dynamical phenomenon is displayed in the synchronization manifold. On the other hand, many existing numerical results always show successful parameter identification. In order to clarify the mechanism inducing such a seeming paradox, we perform a more delicate argument by adopting the LaSalle invariance principle [19] as follows. Similar to [1], set a Lyapunov function candidate by

$$
V(\boldsymbol{e}, \boldsymbol{\epsilon}, \boldsymbol{q})=\frac{1}{2} \sum_{i=1}^{n} e_{i}^{2}+\frac{1}{2} \sum_{i=1}^{n} \sum_{j=1}^{m} \frac{1}{\delta_{i j}}\left(q_{i j}-p_{i j}\right)^{2}+\frac{1}{2} \sum_{i=1}^{n} \frac{1}{r_{i}}\left(\epsilon_{i}+L\right)^{2} .
$$


Then, the derivative of the function $V(\boldsymbol{e}, \boldsymbol{\epsilon}, \boldsymbol{q})$ along with the coupled systems (11) and (3) could be estimated by

$$
\dot{V}(\boldsymbol{e}, \boldsymbol{\epsilon}, \boldsymbol{q}) \leqslant(n l-L) \sum_{i=1}^{n} e_{i}^{2} .
$$

Here, it should be pointed out that $l$ is not the locally Lipschitiz constant of the function $F_{i}(\boldsymbol{x}, \boldsymbol{p})$ but the uniformly Lipschitiz constant since the bounded property of the trajectory $\boldsymbol{y}(t)$ generated by the newly response system (3) are not confirmed but pending for confirmation yet.

Now, we contend that $\boldsymbol{e}(t), \boldsymbol{\epsilon}(t)$, and $\boldsymbol{q}(t)$ are bounded for all $t \geqslant t_{0}$, where $t_{0}$ is the initial time. Indeed, one of the three variables is supposed to be unbounded on $\left[t_{0},+\infty\right)$, so that $V(\boldsymbol{e}(t), \boldsymbol{\epsilon}(t), \boldsymbol{q}(t))$ is also unbounded on $\left[t_{0},+\infty\right)$ according to (10). On the other hand, $V(\boldsymbol{e}(t), \boldsymbol{\epsilon}(t), \boldsymbol{q}(t)) \leqslant V\left(\boldsymbol{e}\left(t_{0}\right), \boldsymbol{\epsilon}\left(t_{0}\right), \boldsymbol{q}\left(t_{0}\right)\right)$ simply due to $\dot{V}(\boldsymbol{e}, \boldsymbol{\epsilon}, \boldsymbol{q}) \leqslant 0$ for sufficiently large $L$. This contradiction thus implies the bounded property of $\boldsymbol{e}(t), \boldsymbol{\epsilon}(t)$, and $\boldsymbol{q}(t)$ for all $t \geqslant t_{0}$.

Therefore, in light of the LaSalle invariance principle, the trajectory $(\boldsymbol{e}(t), \boldsymbol{\epsilon}(t), \boldsymbol{q}(t))$ initiating from any location in the phase plane will eventually approach the largest invariant set $\mathcal{M}$ contained in the set

$$
\mathcal{E}=\{(\boldsymbol{e}, \boldsymbol{\epsilon}, \boldsymbol{q}) \mid \dot{V}(\boldsymbol{e}, \boldsymbol{\epsilon}, \boldsymbol{q})=0\}
$$

Then, the main concern becomes how to make a clear description of the invariant set $\mathcal{M}$ ever contained in the set $\mathcal{E}$ with respect to systems (11) and (3). To this end, a combination of systems (11) and (3) yields

$$
\begin{aligned}
& \dot{e}_{i}=\dot{x}_{i}-\dot{y}_{i}=c_{i}(\boldsymbol{x})-c_{i}(\boldsymbol{y})+\sum_{j=1}^{m} p_{i j} f_{i j}(\boldsymbol{x})-\sum_{j=1}^{m} q_{i j} f_{i j}(\boldsymbol{y})-\epsilon_{i}\left(y_{i}-x_{i}\right) \\
& =c_{i}(\boldsymbol{x})-c_{i}(\boldsymbol{y})+\sum_{j=1}^{m}\left[p_{i j} f_{i j}(\boldsymbol{x})-q_{i j} f_{i j}(\boldsymbol{x})\right]+\sum_{j=1}^{m}\left[q_{i j} f_{i j}(\boldsymbol{x})-q_{i j} f_{i j}(\boldsymbol{y})\right]-\epsilon_{i}\left(y_{i}-x_{i}\right) .
\end{aligned}
$$

Also, notice that $\dot{V}(\boldsymbol{e}, \boldsymbol{\epsilon}, \boldsymbol{q})=0$ implies $\boldsymbol{e}=\boldsymbol{x}-\boldsymbol{y}=\mathbf{0}, \dot{\epsilon}_{i}(t) \equiv 0$, and $\dot{q}_{i j}(t) \equiv 0$. It thus follows from (11) that, for every orbit $(\boldsymbol{e}(t), \boldsymbol{\epsilon}(t), \boldsymbol{q}(t)) \in \mathcal{E}$ of the coupled systems,

$$
\sum_{j=1}^{m}\left[p_{i j}-q_{i j}(t)\right] f_{i j}(\boldsymbol{x}(t))=0
$$


where each $q_{i j}(t)$ is identical to some constant $q_{i j}^{*}$. And the largest invariant set contained in $\mathcal{E}$ with respect to systems (10) and (3) is

$$
\mathcal{M}=\left\{(\boldsymbol{e}, \boldsymbol{\epsilon}, \boldsymbol{q}) \mid \boldsymbol{e}=\boldsymbol{x}-\boldsymbol{y}=\mathbf{0}, \epsilon_{i}=\epsilon_{i}^{*}, q_{i j}=q_{i j}^{*}\right\}
$$

where $\boldsymbol{x}=\boldsymbol{x}(t)$ is the synchronized orbit, or mathematically say, the positive limit set of the driving signal. Thus, the question becomes: "Is each $q_{i j}^{*}$ surely equal to $p_{i j}$ ?" From (12), the answer to this question is theoretically positive provided that [LIM]: for any given $i,\left\{f_{i j}(\boldsymbol{x}), j=1,2, \ldots, m\right\}$ are linearly independent on the synchronized orbit $\boldsymbol{x}=\boldsymbol{x}(t)$ in the synchronization manifold.

For an accurate definition of linearly independent or linearly dependent functions, refer to [20]. Also, it is valuable to mention that two functions might be linearly independent in a domain but linearly dependent in some subset contained in this domain. For example, functions $g_{1}(s, u)=s$ and $g_{2}(s, u)=u^{2}$ are obviously linearly independent in $\mathbb{R}^{2}$ but they are linearly dependent in a parabola-like subset $\mathcal{S}_{\mu}=\left\{(s, u) \in \mathbb{R}^{2} \mid s=\mu u^{2}\right\} \subset \mathbb{R}^{2}$ for some nonzero constant $\mu$.

If hypothesis [LIM] is not satisfied, for some $i=i_{0}$, either there exist two nonzero functions $f_{i_{0} j_{1}}(\boldsymbol{x})$ and $f_{i_{0} j_{2}}(\boldsymbol{x})$ linearly dependent on the orbit $\boldsymbol{x}(t)$ in the synchronization manifold, or simply $f_{i_{0} j_{1}}(\boldsymbol{x}(t)) \equiv 0$. We focus on the former case since failure of parameter identification could be easily illustrated in the latter case. Accordingly, $f_{i_{0} j_{1}}(\boldsymbol{x}(t))=$ $c f_{i_{0} j_{2}}(\boldsymbol{x}(t))$ for some nonzero constant $c$, which at most implies that $\left[p_{i_{0} j_{1}}-q_{i_{0} j_{1}}(t)\right]+$ $c\left[p_{i_{0} j_{2}}-q_{i_{0} j_{2}}(t)\right]=0$. Clearly, although $q_{i_{0} j_{1}}(t)$ and $q_{i_{0} j_{2}}(t)$ are, respectively, identical to some constants $q_{i_{0} j_{1}}^{*}$ and $q_{i_{0} j_{2}}^{*}$, it is not certain that $q_{i_{0} j_{1}}^{*}=p_{i_{0} j_{1}}$ and $q_{i_{0} j_{2}}^{*}=p_{i_{0} j_{2}}$. Actually, they are totally distinct in most cases. Therefore, parameter identification might always be failed if hypothesis [LIM] is not strictly satisfied in the design of driving-and-response systems.

Next, by virtue of the argument performed above, the reason why parameter identification fails in the three examples given in the previous section is expatiated as follows.

For the driving-and-response systems (44) and (5) with specific parameters, the synchronized orbit $\boldsymbol{x}^{*}(t)=\left(x_{1}^{*}(t), x_{2}^{*}(t), x_{3}^{*}(t)\right)^{T}$ in the synchronization manifold, as shown in Fig.1, is a globally asymptotical equilibrium $E=\boldsymbol{x}^{*}(t)=(6.8313,6.8313,1.6667)^{T}$. 
Substitution of (4) into (12) gives

$$
\left[p_{1}-q_{1}(t)\right]\left[x_{2}^{*}(t)-x_{1}^{*}(t)\right]=0,\left[p_{2}-q_{2}(t)\right] x_{1}^{*}(t)=0, \quad\left[p_{3}-q_{3}(t)\right] x_{3}^{*}(t)=0,
$$

where each $q_{i}(t)$ is identical to some constant $q_{i}^{*}$ in the invariant set $\mathcal{M}(i=1,2,3)$. According to [20], each $x_{i}^{*}(t)(\neq 0)$ is linearly independent and $x_{2}^{*}(t)-x_{1}^{*}(t)(\equiv 0)$ is linearly dependent. This implies that $q_{2,3}^{*}$ is identical to $p_{2,3}$ but $q_{1}^{*}$ is not necessarily identical to $p_{1}$. Therefore, $q_{1}(t)$, though obeying the updating law, will not be surely convergent to $p_{1}$. This illustrates the reason why parameter identification succeeds for $q_{2,3}$ but always fails for $q_{1}$ as shown in Fig.2. However, when the synchronized orbit $\boldsymbol{x}^{*}(t)$ with the classical parameters is chaotic, $x_{2}^{*}(t)-x_{1}^{*}(t)$ is nonzero and thus is linearly independent, which satisfies hypothesis $[\mathrm{LIM}]$. Hence, $q_{1}(t)$ will be convergent to $p_{1}$ almost surely, as is shown by many existing numerical results. In addition, when the synchronized orbit $\boldsymbol{x}^{*}(t)$ unfortunately becomes the unstable equilibrium of the original chaotic systems, $x_{2}^{*}(t)-x_{1}^{*}(t)$ is still identical to zero violating hypothesis [LIM]. So, $q_{1}(t)$ still will not be surely convergent to the accurate value of $p_{1}$ in such a case.

For the coupled systems (6) and (7), the orbit $\boldsymbol{x}^{*}(t)$ in the synchronization manifold, as mentioned above, is designed to be some kind of stable periodic orbit. Its projection into the $x_{1}-x_{2}$ plane, which seems like an ellipse, could be approximately expressed by the formula

$$
\frac{\left(x_{1} \cos (0.9026)+x_{2} \sin (0.9026)\right)^{2}}{23.44^{2}}+\frac{\left(-x_{1} \sin (0.9026)+x_{2} \cos (0.9026)\right)^{2}}{7.19^{2}}-1=0 .
$$

Also, substitution of (6) into (12) yields

$$
\begin{aligned}
& {\left[p_{1}-q_{1}(t)\right] \frac{\left(x_{1}(t) \cos (0.9026)+x_{2}(t) \sin (0.9026)\right)^{2}}{23.44^{2}}+} \\
& {\left[-p_{2}+q_{2}(t)\right]\left\{\frac{\left(-x_{1}(t) \sin (0.9026)+x_{2}(t) \cos (0.9026)\right)^{2}}{7.19^{2}}-1\right\}=0 .}
\end{aligned}
$$

Thus, as long as the complete synchronization between systems (6) and (7) is achieved, the orbit $\boldsymbol{x}(t)$, as well as $\boldsymbol{y}(t)$, will approximately approach the stable periodic orbit $\boldsymbol{x}^{*}(t)$. Then, both functions

$$
\frac{\left(x_{1}^{*}(t) \cos (0.9026)+x_{2}^{*}(t) \sin (0.9026)\right)^{2}}{23.44^{2}} \text { and } \frac{\left(-x_{1}^{*}(t) \sin (0.9026)+x_{2}^{*}(t) \cos (0.9026)\right)^{2}}{7.19^{2}}-1
$$


are approximately linearly dependent. This, according to the argument performed above, means that both $q_{1}$ and $q_{2}$ are not suitable for parameter identification, as is verified by the simulation results in Fig.4.

Unlike the steady dynamics exhibiting in synchronization manifold in the previous two examples, the synchronized orbit $\boldsymbol{x}^{*}(t)$ generated by the driving system (으) is deliberately designed to be chaotic in the sense of possessing positive Lyapunov exponent. Analogously, substitution of (8) into (12) produces

$$
\left[p_{3}-q_{3}(t)\right] x_{3}(t)+\left[p_{4}-q_{4}\right] x_{3}(t)\left\{1+\left[x_{4}(t)\right]^{3}\right\}=0 .
$$

It is obvious that functions $x_{3}$ and $x_{3}\left(1+x_{4}^{3}\right)$ are linearly independent in the whole phase plane $\mathbb{R}^{4}$; nevertheless, they are approximately linearly dependent on the synchronized orbit $\boldsymbol{x}^{*}(t)$ because the cubic term $\left[x_{4}^{*}(t)\right]^{3}$ is almost equal to zero as time $t$ is sufficiently large (see Fig.7). Thus, this illustrates the reason why $q_{3}$ and $q_{4}$ initiating from a mass of points will not be convergent to the accurate values of $p_{3}$ and $p_{4}$, respectively, in concrete numerical simulations.

In addition, consider a case that parameter $b$ in both systems (8) and (9) is selected to be zero instead of 0.1. This case could be regarded as a very special example where parameter identification may fail in spite of existence of chaos. In such an example, because of $x_{4}^{*}(t) \equiv 0$, functions $x_{3}$ and $x_{3}\left(1+x_{4}^{3}\right)$ are definitely linearly dependent on the corresponding orbit $\boldsymbol{x}^{*}(t)$, which violates hypothesis [LIM]. Therefore, $q_{3}$ and $q_{4}$ can not be utilized to identify the parameters $p_{3}$ and $p_{4}$. In a word, chaotic property of synchronized orbit in synchronization manifold does not always guarantee a success in parameter identification.

Remark 3.1 In the last two examples, those functions on the orbits in the synchronization manifold are approximately linearly dependent. Rigorously, they are still linearly independent in a mathematical sense, so that parameter identification might be theoretically achieved for $q_{i}$ correspondingly with $p_{i}$. However, in real application, discretization techniques, such as the Runge-Kutta scheme and the Euler scheme, are always taken into account in solving the coupled continuous differential systems. Thus, owing to the precision limit, it is unavoidable that dynamics produced by the discretized system may not 
be completely consistent with the true dynamics generated by the original system. It is the approximate dependence of those functions that poses some trap of local critical point for $q_{i}$ and that leads to a failure of parameter identification in the last two examples. Therefore, not only rigorous linear-dependence of those functions with parameters pending for identification on the synchronized orbit but also approximate linear-dependence on the synchronized orbit should be always avoided whenever adaptive synchronization techniques are used in practical parameter estimation and chaos communication.

\section{Complete Synchronization without Globally Lipschitz Con- dition}

In the previous section, it is pointed out that hypothesis $[\mathrm{LIM}]$ is indispensable for a successful parameter identification. In addition, the uniform Lipschitz condition for $\boldsymbol{F}(\boldsymbol{x}, \boldsymbol{p})$ is also important in the argument performed above for obtaining a non-positive property of $\dot{V}(\boldsymbol{e}, \boldsymbol{\epsilon}, \boldsymbol{q})$. As a matter of fact, this uniform condition could be loosed if the bounded property of the response system (3) could be priorly estimated. However, this prior estimation could not directly follow from the bounded property of driving system (11) since dynamics of its response system with coupling term might be completely different from the driving system which is, though, supposed to be bounded in advance. Then it poses a question: "Other than the above coupling technique and uniform Lipschitz condition, under what kind of coupling methods and conditions on $\boldsymbol{F}(\boldsymbol{x}, \boldsymbol{p})$ can one obtain a successful parameter identification rigorously?"

To this end, it is first assumed [HPT]: each $F_{i}(\boldsymbol{x}, \boldsymbol{p})$ is homogeneous polynomial with degree no more than two with respect to $\boldsymbol{x}$. As a matter of fact, a large quantities of nonlinear systems does not satisfy globally Lipschitz condition but are consistent with this assumption [HPT], such as the Lorenz system and the Chen's system.

Next, notice that

$$
\begin{aligned}
2 y_{k} y_{j}-2 x_{k} x_{j} & =\left(y_{k}-x_{k}\right)\left(y_{j}+x_{j}\right)+\left(y_{j}-x_{j}\right)\left(y_{k}+x_{k}\right) \\
& =2 e_{k} e_{j}+2 x_{j} e_{k}+2 x_{k} e_{j}
\end{aligned}
$$


for arbitrary $k$ and $j$. Then, it is easy to verify that each $e_{i}\left[F_{i}(\boldsymbol{y}, \boldsymbol{p})-F_{i}(\boldsymbol{x}, \boldsymbol{p})\right]$ can be written as a homogeneous polynomial with degree no more than three with respect to $\boldsymbol{e}=\boldsymbol{y}-\boldsymbol{x}$ if assumption [HPT] holds.

Reasonably, the driving signal $\boldsymbol{x}(t)$ generated by system (11) is supposed to be bounded in advance. In order to obtain a rigorous synchronization in the system where the nonlinearity only satisfies assumption [HPT], we re-designed the response system as:

$$
\begin{aligned}
& \dot{\boldsymbol{y}}=\boldsymbol{F}(\boldsymbol{y}, \boldsymbol{q})+\boldsymbol{\epsilon} \cdot \boldsymbol{e}+\boldsymbol{\omega} \cdot \boldsymbol{e}^{3}, \\
& \dot{\epsilon}_{i}=-r_{i} e_{i}^{2}, \quad \dot{\omega}_{i}=-s_{i} e_{i}^{4}, \\
& \dot{q}_{i j}=-\delta_{i j} e_{i} f_{i j}(\boldsymbol{y}),
\end{aligned}
$$

where $\boldsymbol{\omega} \cdot \boldsymbol{e}^{3}=\left(\omega_{1} e_{1}^{3}, \omega_{2} e_{2}^{3}, \cdots, \omega_{n} e_{n}^{3}\right)^{T}$, each $s_{i}$ is arbitrarily positive constant, and other states and parameters are the same as those defined in (3).

Set a Lyapunov function candidate by $H(\boldsymbol{e}, \boldsymbol{\epsilon}, \boldsymbol{\omega}, \boldsymbol{q})=\frac{1}{2} \sum_{i=1}^{n} e_{i}^{2}+\frac{1}{2} \sum_{i=1}^{n} \sum_{j=1}^{m} \frac{1}{\delta_{i j}}\left(q_{i j}-p_{i j}\right)^{2}+\frac{1}{2} \sum_{i=1}^{n} \frac{1}{r_{i}}\left(\epsilon_{i}+M\right)^{2}+\frac{1}{2} \sum_{i=1}^{n} \frac{1}{s_{i}}\left(\omega_{i}+N\right)^{2}$. Thus, the derivative of this function along with the coupled systems (11) and (13) yields

$$
\dot{H}(\boldsymbol{e}, \boldsymbol{\epsilon}, \boldsymbol{\omega}, \boldsymbol{q})(t)=\sum_{i=1}^{n} e_{i}(t)\left[F_{i}(\boldsymbol{y}(t), \boldsymbol{p})-F_{i}(\boldsymbol{x}(t), \boldsymbol{p})\right]-\sum_{i=1}^{n} M e_{i}^{2}(t)-\sum_{i=1}^{n} N e_{i}^{4}(t),
$$

where both $M$ and $N$ are positive numbers. By virtue of the conclusion on each $e_{i}\left[F_{i}(\boldsymbol{y}, \boldsymbol{p})-\right.$ $\left.F_{i}(\boldsymbol{x}, \boldsymbol{p})\right]$ obtained above, the elementary inequality

$$
e_{i} e_{j} e_{k} \leqslant \frac{1}{6} \sum_{l=i, j, k}\left(e_{l}^{2}+e_{l}^{4}\right)
$$

and the assumed bounded property of the driving signal $\boldsymbol{x}(t)$ and parameter set $\mathcal{U}$, we can obtain that $\dot{H}(\boldsymbol{e}, \boldsymbol{\epsilon}, \boldsymbol{\omega}, \boldsymbol{q})(t) \leqslant 0$ for sufficiently large numbers $M$ and $N$.

By using the similar argument performed in the previous section, we can easily prove that every trajectory generated by the coupled systems (11) and (13) is not only bounded for all $t \geqslant t_{0}$ but also approaching the largest invariant set contained in

$$
\mathcal{E}^{\prime}=\{(\boldsymbol{e}, \boldsymbol{\epsilon}, \boldsymbol{\omega}, \boldsymbol{q}) \mid \dot{H}(\boldsymbol{e}, \boldsymbol{\epsilon}, \boldsymbol{\omega}, \boldsymbol{q})=0\}
$$


with respect to these coupled systems. More precisely, the largest invariant set becomes

$$
\mathcal{M}^{\prime}=\left\{(\boldsymbol{e}, \boldsymbol{\epsilon}, \boldsymbol{\omega}, \boldsymbol{q}) \mid \boldsymbol{e}=\mathbf{0}, \epsilon_{i}=\epsilon_{i}^{*}, \omega_{i}=\omega_{i}^{*}, q_{i j}=q_{i j}^{*}\right\},
$$

where $\epsilon_{i}^{*}, \omega_{i}^{*}$, and $q_{i j}^{*}$ are some constants dependent on the initial values of the coupled systems. Furthermore, to achieve an accurate parameter identification between systems (1) and (13), hypothesis [LIM] should be still adopted. Then, the above performed argument could be concluded as the following proposition.

Proposition 4.1 If assumptions [LIM] and [HPT] on $\boldsymbol{F}(\boldsymbol{x}, \boldsymbol{p})$ are satisfied, the complete synchronization between driving system (11) and its response system (13) could be surely achieved, and the parameter identification could be accurately realized in a mathematical sense.

Remark 4.2 As mentioned above, in numerical experiment and even in real application, not only hypothesis [LIM] should be strictly satisfied but also the approximate lineardependence attributed to precision limit should be avoided.

Remark 4.3 Assumption [HPT] on $\boldsymbol{F}(\boldsymbol{x}, \boldsymbol{p})$ could be further generalized to some other case where globally Lipschitz condition is not fulfilled. For instance, one could further consider the system where the degree of the homogeneous polynomials is larger than two, or some of those polynomials are non-homogeneous. However, additional coupling terms (e.g. $e^{2 v+1}, v=2,3, \cdots$ ) should be added into the response systems in order to obtain a successful synchronization and parameter identification in a rigorous sense.

Remark 4.4 As a matter of fact, nonlinearities in the previous three examples are not globally Lipschitz but polynomial. Theories and coupling techniques (i.e. Proposition 4.1 and Remark 4.3) proposed in this section should be utilized to deal with those systems for obtaining a successful synchronization and parameter identification.

\section{Parameter Identification in Systems with Time-Delay}

Time-delay, as an omnipresent phenomenon, can not be neglected in practice. So, in this section, complete synchronization and parameter identification in time-delayed systems 
via adaptive coupling techniques are further investigated. For simplicity, consider a onedimensional driving system:

$$
\dot{x}(t)=a f(x(t))+b g(x(t-\tau))
$$

where $\tau \geqslant 0$ is a time-delay, $a$ and $b$ are parameters pending for identification, and functions $f$ and $g$ are assumed to be globally Lipschitz continuous with Lipschitz constants $k_{f}$ and $k_{g}$, respectively. Given driving signal $x(t)$ generated by system (14), the response system is designed as

$$
\begin{aligned}
& \dot{y}(t)=\alpha(t) f(y(t))+\beta(t) g(y(t-\tau))+\eta(t) e(t)+\omega(t) e(t-\delta), \\
& \dot{\alpha}(t)=-f(y(t)) e(t), \quad \dot{\beta}(t)=-g(y(t-\tau)) e(t), \\
& \dot{\eta}(t)=-e^{2}(t), \quad \dot{\omega}(t)=-e(t) e(t-\delta),
\end{aligned}
$$

where $\delta \geqslant 0$ is a time-delay induced by coupling term, error dynamics $e(t)=y(t)-x(t)$. The initial conditions for coupled system (14) and (15) are chosen as $e=\phi, \alpha=A, \beta=B$, $\eta=E, \omega=W \in \mathcal{C} \triangleq \mathbb{C}([-\max \{\tau, \delta\}, 0], \mathbb{R})$, in which $\mathcal{C}$ denotes the sets of all continuous functions from $[-\max \{\tau, \delta\}, 0]$ to $\mathbb{R}$.

Set a Lyapunov functional candidate by

$$
\begin{aligned}
\mathcal{V}(\phi, A, B, E, W)= & \frac{1}{2} \phi^{2}(0)+\frac{1}{2}[A(0)-a]^{2}+\frac{1}{2}[B(0)-b]^{2}+\frac{1}{2}[E(0)+L]^{2} \\
& +\frac{1}{2}[W(0)+M]^{2}+\left\{\int_{-\tau}^{0}+\int_{-\delta}^{0}\right\} \phi^{2}(s) \mathrm{d} s
\end{aligned}
$$

where $L, M$ are some proper positive constant. Then, the derivative of $\mathcal{V}$ along with coupled systems (14) and (15) could be estimated by

$$
\begin{aligned}
\dot{\mathcal{V}}(\phi, A, B, E, W) \leqslant & \left(a k_{f}+2-\frac{L}{2}\right) \phi^{2}(0)+b k_{g} \cdot|\phi(0)| \cdot|\phi(-\tau)|-\phi^{2}(-\tau) \\
& -\frac{L}{2} \phi^{2}(0)-M \phi(0) \phi(-\delta)-\phi^{2}(-\delta) .
\end{aligned}
$$

Clearly, $\dot{\mathcal{V}}(\phi, A, B, E, W)$ becomes non-positive provided $L>\max \left\{\frac{M^{2}}{2}, \frac{b^{2} k_{g}^{2}}{2}+2 a k_{f}+\right.$ $4\}$. By using a similar argument performed above, we can conclude that every trajectory $\left(e_{t}(\phi), \alpha_{t}(A), \beta_{t}(B), \eta_{t}(E), \omega_{t}(W)\right)$, starting from arbitrary initial condition, is surely bounded for all $t \geqslant-\max \{\tau, \delta\}$. 
Then, according to the invariance principle for the systems with time-delay [21, every trajectory, as time tends towards positive infinity, approaches the largest invariant set $\widetilde{\mathcal{M}}$ contained in

$$
\widetilde{\mathcal{E}}=\{(\phi, A, B, E, W) \in \underbrace{\mathcal{C} \times \cdots \times \mathcal{C}}_{5} \mid \phi(0)=\phi(-\tau)=\phi(-\delta)=0\}
$$

with respect to coupled systems (14) and (15)). This further implies that the first component of each element in $\widetilde{\mathcal{M}}$ is identical to zero (i.e. $\phi \equiv 0$ ) and the others are some constant functions (i.e. $A \equiv A^{*}, B \equiv B^{*}, E \equiv E^{*}$, and $W \equiv W^{*}$ ). The accurate values of these constant functions rest on the initial conditions of coupled systems (14) and (15).

Parameter identification could be achieved if both equations $A^{*}=a$ and $B^{*}=b$ are valid. However, these equations are not always tenable although $\phi \equiv 0$ indicates a successful complete synchronization between systems (14) and (15). As a matter of fact, subtraction of (14) from the first equation in (15) yields, in $\widetilde{\mathcal{M}}$,

$$
\begin{aligned}
0 & =\alpha(t) f(y(t))-a f(x(t))+\beta(t) g(y(t-\tau))-b g(x(t-\tau)) \\
& =[\alpha(t)-a] f(x(t))+[\beta(t)-b] g(x(t-\tau)) \\
& =\left[A^{*}-a\right] f(x(t))+\left[B^{*}-b\right] g(x(t-\tau)),
\end{aligned}
$$

which follows from $e(t)=y(t)-x(t) \equiv 0$ in $\widetilde{\mathcal{M}}$. Now, it is clear that when functions $f(x(t))$ and $g(x(t-\tau))$, on the synchronized orbit $x(t)$ in the synchronization manifold, are linearly dependent, both $A^{*}=a$ and $B^{*}=b$ are not certainly tenable. More concretely, (i) when the driving signal asymptotically tends towards some equilibrium $x(t) \equiv x^{*}$ of system (14), two constant functions $f(x(t)) \equiv f\left(x^{*}\right)$ and $g(x(t-\tau)) \equiv g\left(x^{*}\right)$ becomes linearly dependent so that parameter identification for $a$ and $b$ will be almost surely failed; (ii) when the synchronized orbit $x(t)$ is periodic with period $\tau$ and both functions $f$ and $g$ are linearly dependent in $\mathbb{R}$, parameter identification also will be failed; (iii) when $x(t$ ) is chaotic, parameter identification will be achieved in a mathematical sense for non-constant differential functions $f$ and $g$, and even for $f=g$ (see an example shown in Fig.8(a) where both $f$ and $g$ are taken as sinusoid functions). However, for case (iii), parameter identification also might be failed in numerical simulation or in real application. For example, in spite of chaotic property of $x(t)$, it is likely that $x(t) \approx x(t-\tau)$ with a small 
time-delay or that fluctuation of $x(t)$ seems to be relatively steady in a macro scale. These extraordinary cases may lead to approximate linear-dependence between functions $f(x(t))$ and $g(x(t-\tau))$, which thus results in failure of parameter identification in numerical simulation. See an illustrative example in Fig.8 (b). In addition, $\tau=0$ could be regarded as a special case where parameter identification is always failed provided that functions $f$ and $g$ are linearly dependent on $x(t)$.

In conclusion, we have the following proposition on synchronization and parameter identification for coupled systems (14) and (15) with time-delay.

Proposition 5.1 The complete synchronization between driving system (14) and its response system (15) could be surely achieved via adaptive coupling techniques. Furthermore, the parameter identification could be accurately realized in a mathematical sense provided that $f(x(t))$ and $g(x(t-\tau))$ are linearly independent on the synchronized orbit $x(t)$ in the synchronization manifold.

Remark 5.2 With analogous arguments but more complicated notations, the results on the driving system (14) could be further generalized to the case where higher dimensional driving systems and multiple parameters identification are taken into account. However, linear-independence of all the functions with unknown parameters on the driving signal is crucial to a successful parameter identification.

\section{Conclusion}

In summary, concrete examples showing possible occurrence of failed parameter identification have been numerically given in the paper. The mechanism inducing this failure has been further rigorously interpreted. It has been pointed out that chaotic property of driving system is not always crucial to achievement of parameter identification either in a mathematical argument or in a numerical experiment. Actually, it is not the chaos but the hypothesis [LIM] that guarantees a successful parameter identification based on adaptive synchronization techniques. However, making good use of chaotic property might easily lead to validity of hypothesis [LIM]. Apart from linear-dependence of functions, approximate linear-dependence of functions with parameters pending for identification on 
the synchronized orbit should be always avoided in numerical simulation and even in real application.

Furthermore, complete synchronization via new adaptive coupling techniques in a class of polynomial systems where nonlinearity is not globally Lipschitz has been theoretically investigated by virtue of the LaSalle invariance principle. Also it has been rigorously verified that every trajectory generated by the coupled systems is bounded. By these derived theoretical results, our newly proposed technique is convinced to be a rigorous and feasible approach for realization of complete synchronization and parameter identification in the Lorenz-like systems. Besides, adaptive coupling techniques are also imported to realize parameter identification in systems with time-delay. Those discussion also shows the great importance of the condition that functions with parameters pending for identification on the synchronized orbit should be linearly independent.

\section{Acknowledgement}

The authors are grateful to the learned referee and Prof. Jiong Ruan for their helpful comments and suggestions. This research was supported by the National Natural Foundation of China Grant No.10501008.

\section{References}

[1] C. Huygens, Philo. Trans. Royal Soc. 4, 937 (1669).

[2] L. M. Pecora and T. L. Carroll, Phys. Rev. Lett. 64, 821 (1990).

[3] E. Ott, C. Grebogi, and J. A. York, Phys. Rev. Lett. 64, 1196 (1990); N. F. Rulkov, M. M. Sushchik, L. S. Tsimring and H. D. I. Abarbanel, Phys. Rev. E. 51, 980 (1995); E. Jr. Rosa, E. Ott, and M. H. Hess, Phys. Rev. Lett. 80, 1642 (1998).

[4] G. Chen, X. Dong, From Chaos to Order: Methodologies, Persperctives and Applications (World Scientific, Singapore, 1998); Handbook of Chaos Control, edited by H. G. Schuster (Wiley-VCH, Weinheim, 1999); S. Boccaletti et al., Phys. Rep. 366, 1 (2002); G. Chen, X. Yu, Chaos Control: Theory and Applications (Springer, Berlin, 2003); E. M. Bollt, Int. J. of Bifur. Chaos, 13, 269 (2003). 
[5] C. S. Zhou and J. Kurths, Phys. Rev. Lett. 88, 230602 (2002); Chaos 13, 401 (2003); W. Lin, Y. He, Chaos 15, 023705 (2005); W. Lin, G. Chen, Chaos 16, 013134 (2006); Z. Chen, W. Lin, J. Zhou, Chaos, in press (2007).

[6] H. Fujisaka and T. Yamada, Prog. Theor. Phys. 69, 32 (1983); T. Yamada and H. Fujisaka, Prog. Theor. Phys. 70, 1240 (1983).

[7] C. Grebogi, E. Ott and J. A. Yorke, Phys. Rev. Lett. 50935 (1983); M. Rosenblum, A. Pikovsky, and J. Kurths, Phys. Rev. Lett. 78, 4193 (1997).

[8] A. Pikovsky, G. Osipov, M. Rosenblum, M. Zaks, and J. Kurths, Phys. Rev. Lett. 79, 47 (1997).

[9] N.F. Rulkov, M.M. Sushchik, L.S. Tsimring, and H.D.I. Abarbanel, Phys. Rev. E 51, 980 (1995); L. Kocarev and U. Parlitz, Phys. Rev. Lett. 76, 1816 (1996).

[10] A. Maybhate and R.E. Amritkar, Phys. Rev. E 59,284 (1999); Phys. Rev. E 61, 6461 (2000).

[11] D.B. Huang, Phys. Rev. Lett. 93, 214101 (2003); Phys. Rev. E 69, 067201 (2004); Phys. Rev. E 71, 037203 (2005); Phys. Rev. E 73, 066204 (2006).

[12] U. Parlitz, Phys. Rev. Lett. 76, 1232 (1996).

[13] M.T. Yassen, Appl. Math. Comput. 135, 113 (2001).

[14] A.S. Hegazi et al., Int. J. Bifucation and Chaos Appl. Sci. Eng. 12, 1579 (2002)

[15] S.H. Chen et al., Phys. Lett. A. 321, 50 (2004).

[16] J. Lu et al., Chaos 15, 043901 (2005).

[17] I.P. Mariño, J. Miguez, Phys. Rev. E 72, 057202 (2005).

[18] J. Zhou et al., Chaos, Solitons and Fractals 27, 905 (2006).

[19] J.P. LaSalle, IRE Trans. Circuit Theory CT-7, 520 (1960); An Invariance Principle in The Theory of Stability, in Differential Equations and Dynamical Systems, edited by J.K. Hale, J.P. LaSalle, (Academic Press, 1967).

[20] A family of real valued functions $\left\{g_{i}(\boldsymbol{x}), i=1,2, \cdots, n\right\}$ are said to be linearly independent in some set $\mathcal{S}$ if and only if $\sum_{i=1}^{n} c_{i} g_{i}(\boldsymbol{x})=0$ (for all $\boldsymbol{x} \in \mathcal{S}$ ) implies each $c_{i}=0$; otherwise, they are said to be linearly dependent. In particular, function $g(\boldsymbol{x}) \equiv 0$ is linearly dependent but single nonzero constant function is linearly independent. 
[21] J.K. Hale, S.V. Lunel, Introduction to Functional Differential Equations in Applied Mathematical Sciences. (Berlin, Germany: Springer-Verlag, 1993); J.K. Hale, Resenhas IME-USP 3, 55 (1997). 


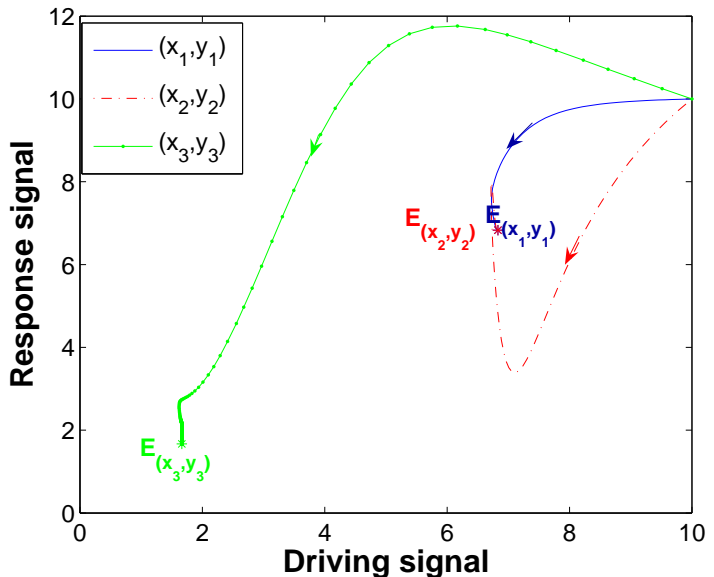

(a)

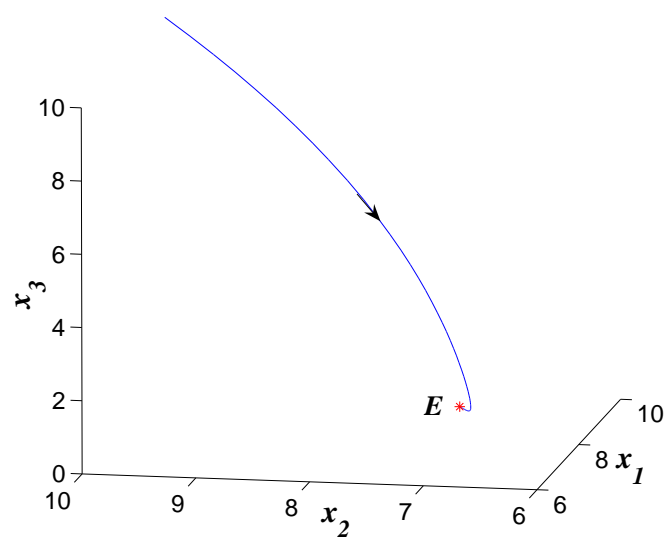

(b)

Fig. 1: (Color online) A successful complete synchronization between the Lorenz system (44) and (5) by means of the adaptive design coupling. Here, system (44) possesses an asymptotically stable equilibrium $E=(6.8313,6.8313,1.6667)^{T}$ instead of the strange attractor. The variation of the driving signal with the response state are shown in (a) and the evolution of response state in the phase plane are depicted in (b). Here, $r_{i}=15, \delta_{i}=2$ and all the initial values are simply chosen as $x_{i}^{0}=y_{i}^{0}=10, q_{i}^{0}=1, \epsilon_{i}^{0}=1(i=1,2,3)$. 

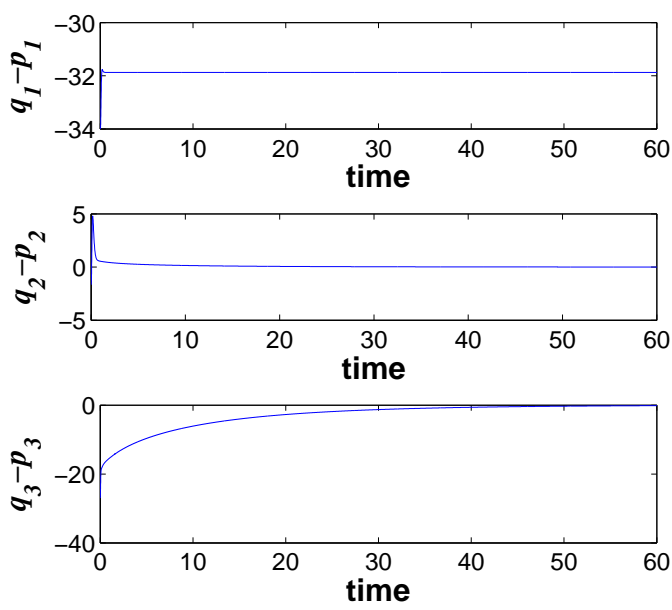

Fig. 2: The variation of the error between the parameters $q_{i}$ and $p_{i}$ with time initiating from 0 to 60 with step-size $0.01(i=1,2,3)$. In particular, $q_{1}$ fails to identify the accurate value of $p_{1}$. All the parameters and initial values for coupling systems are the same as those given in Fig.1. 


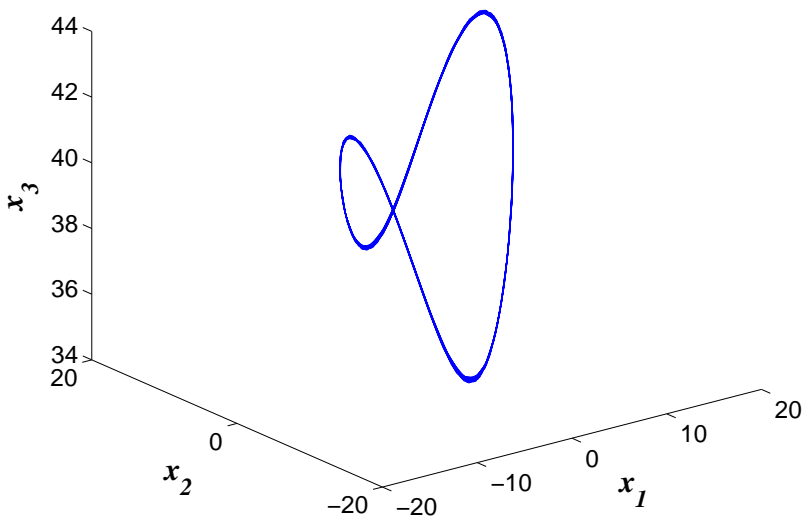

(a)

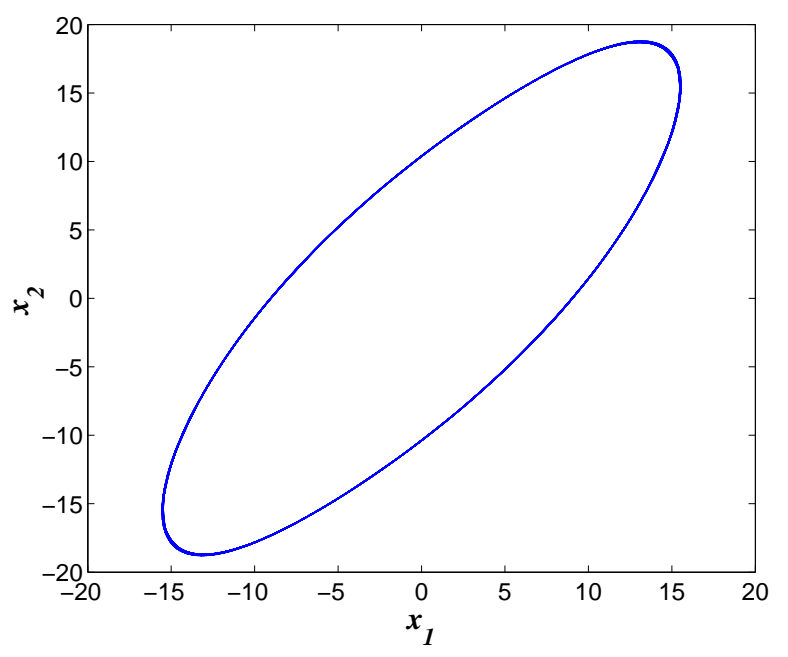

(b)

Fig. 3: The attractive periodic orbit generated by the original Chen's system (system (6) when $\mathcal{Q} \equiv 0$ ). The periodic orbit in the $x_{1}-x_{2}-x_{3}$ phase plane (a) and its projection in the $x_{1}-x_{2}$ plane (b). 

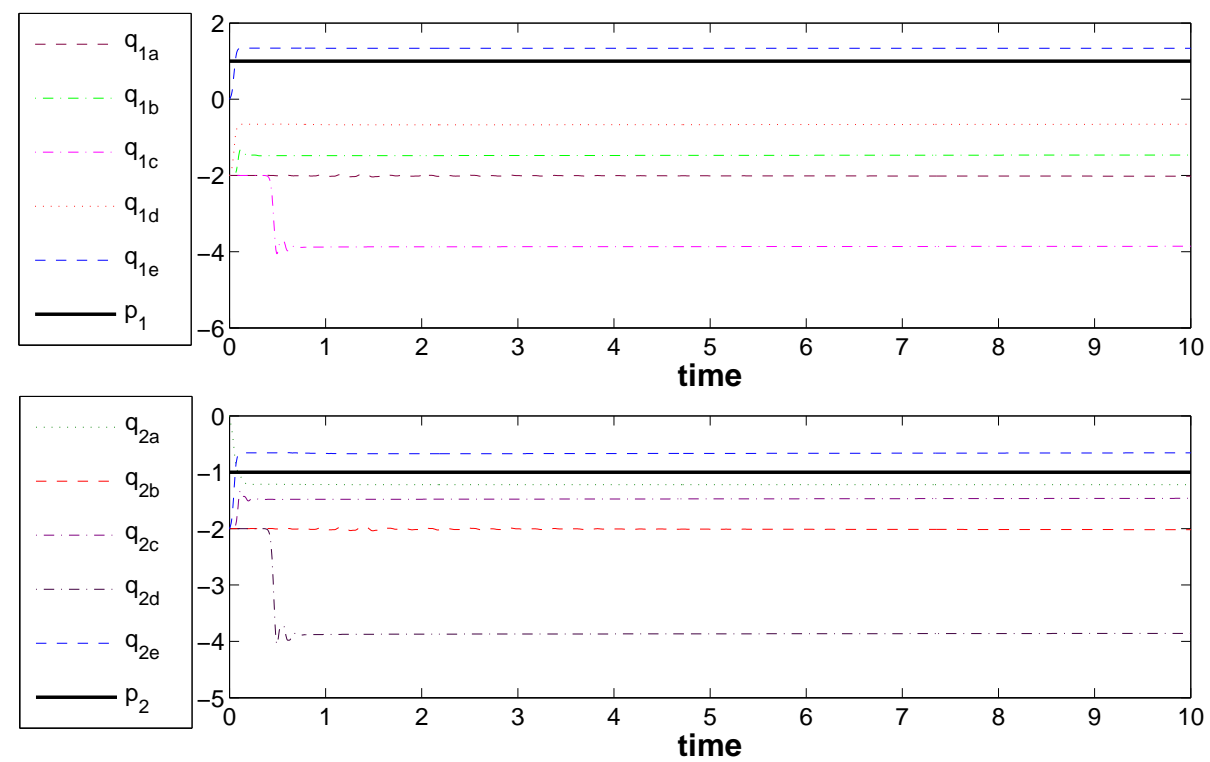

Fig. 4: (Color online) The variation of $q_{i}(i=1,2)$ with time initiating from 0 to 15 with step-size 0.01 when its initial value $q_{i \varrho}$ is differently taken $(\varrho=a, b, c, d, e)$. Here, $r_{j}=2, \delta_{j}=1$ and all the initial values are taken as $x_{j}^{0}=y_{j}^{0}=10, \epsilon_{j}^{0}=1, j=1,2,3$. 


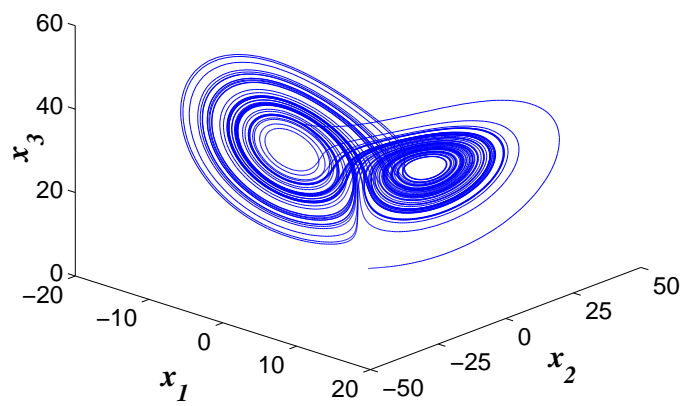

(a)

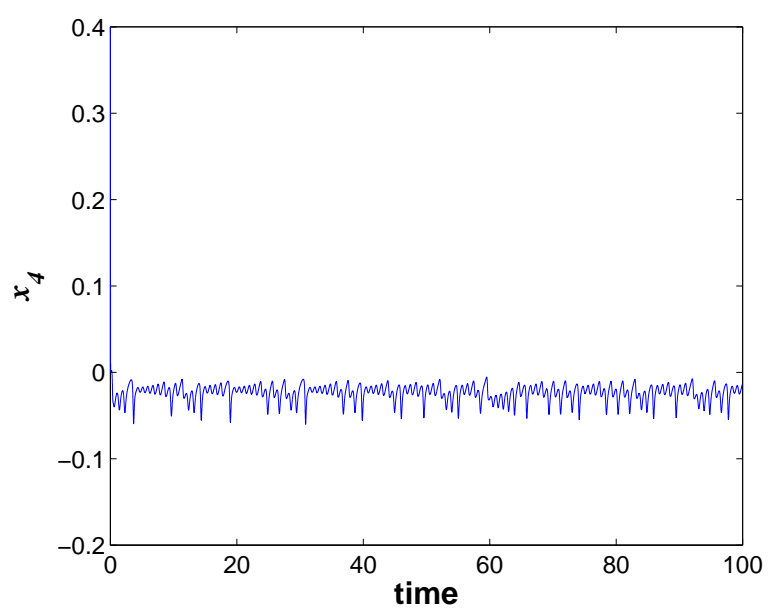

(b)

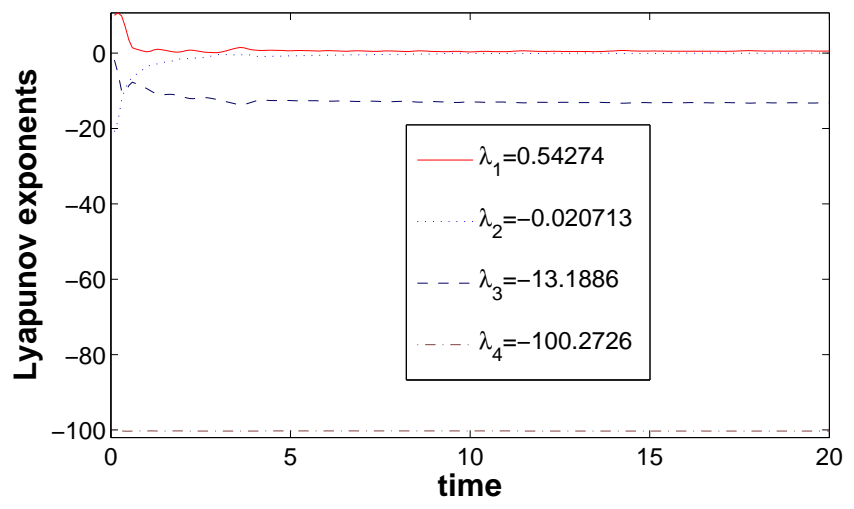

(c)

Fig. 5: (Color online) The strange attractor produced by system (8) are, respectively, plotted in the $x_{1}-x_{2}-x_{3}$ plane (a) and in the time-state- $x_{4}$ plane (b). The chaotic property is verified by the Lyapunov exponent portrait (c), where the largest Lyapunov exponent $\lambda_{1}$ is above zero. 

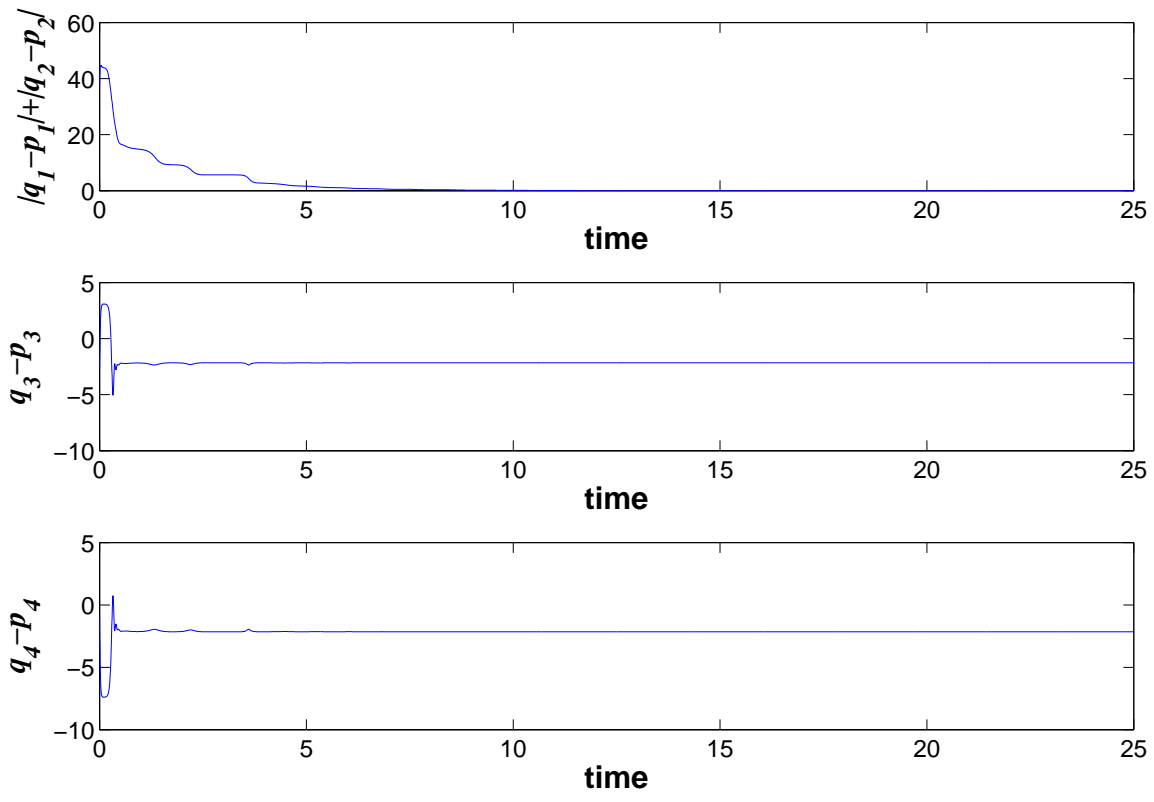

Fig. 6: The variation of the error between the parameters $q_{j}$ and $p_{j}$ with time initiating from 0 to 25 with step-size 0.01 . Indeed, $q_{3,4}$ fails to identify the accurate value of $p_{3,4}$, respectively. Here, $r_{j}=15, \delta_{j}=2$, and all the initial values are taken as $x_{j}^{0}=1, y_{1}^{0}=6, y_{2}^{0}=, y_{3}^{0}=10, y_{4}^{0}=1$, $q_{j}^{0}=0$, and $\epsilon_{j}^{0}=1(j=1,2,3,4)$.
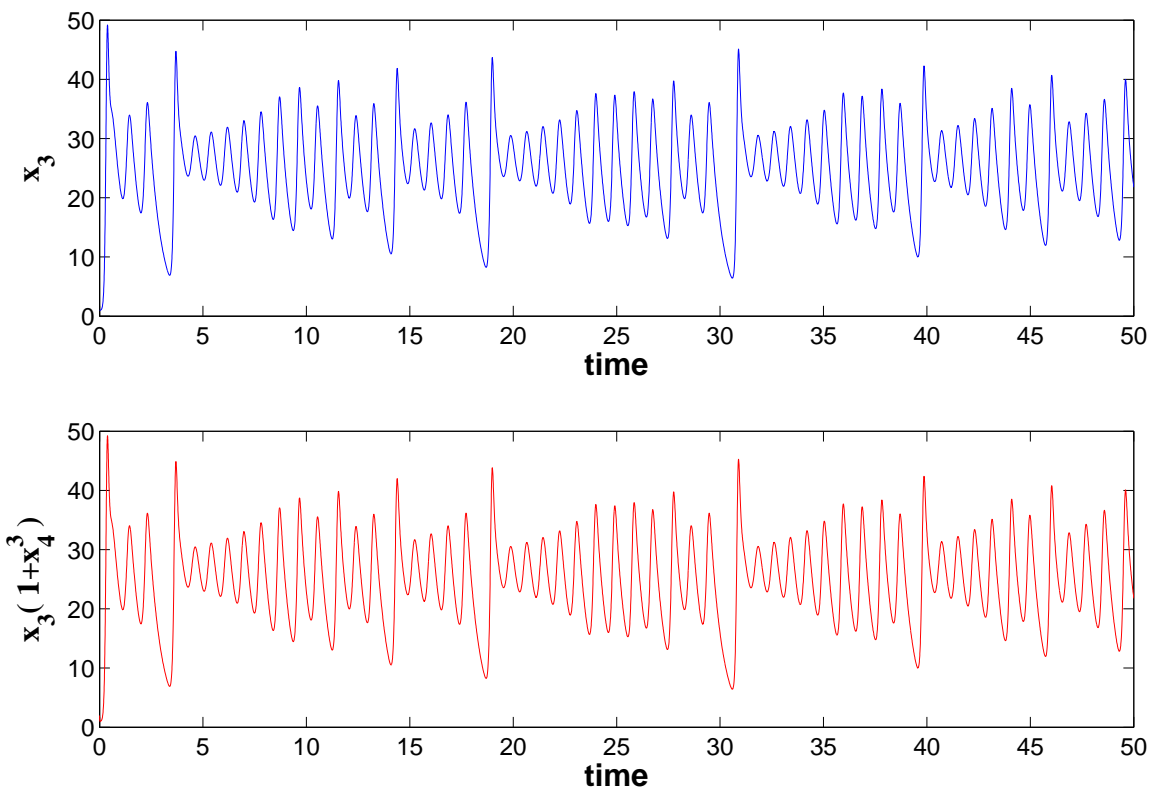

Fig. 7: (Color online) The variation of $x_{3}$ and $x_{3}\left(1+x_{4}^{3}\right)$ on the synchronized orbit $\boldsymbol{x}^{*}(t)$ with time, respectively 

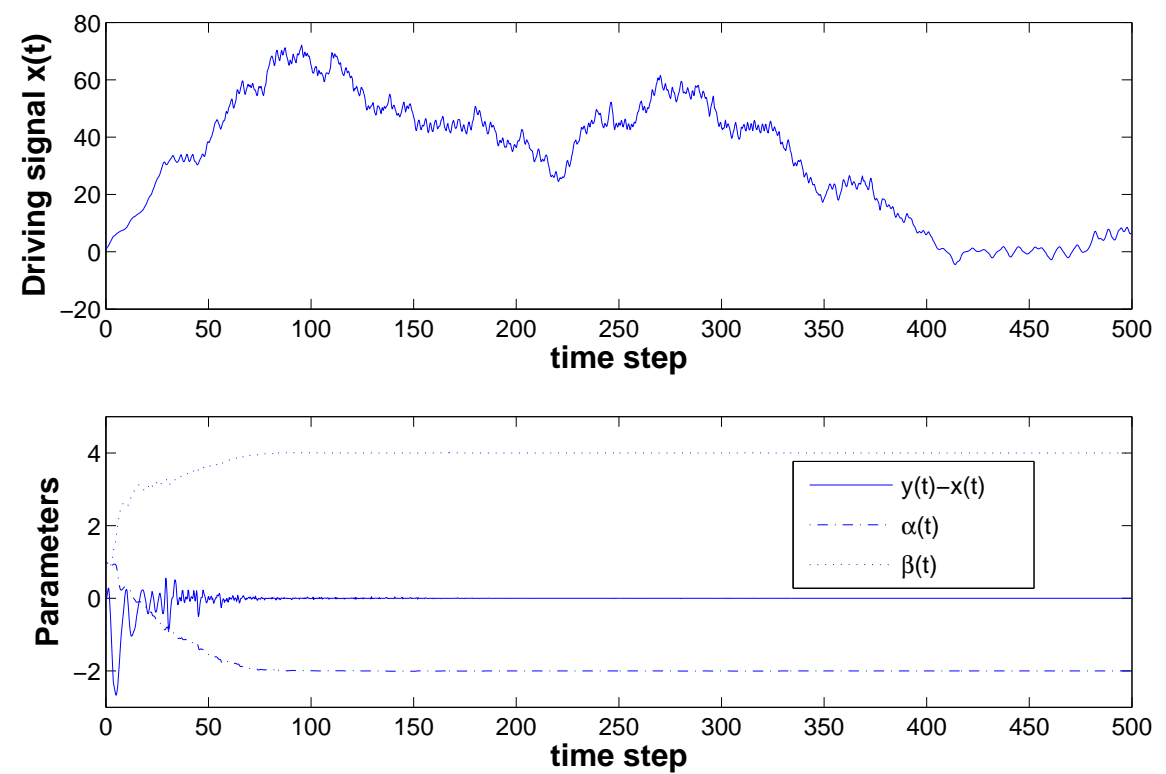

(a)
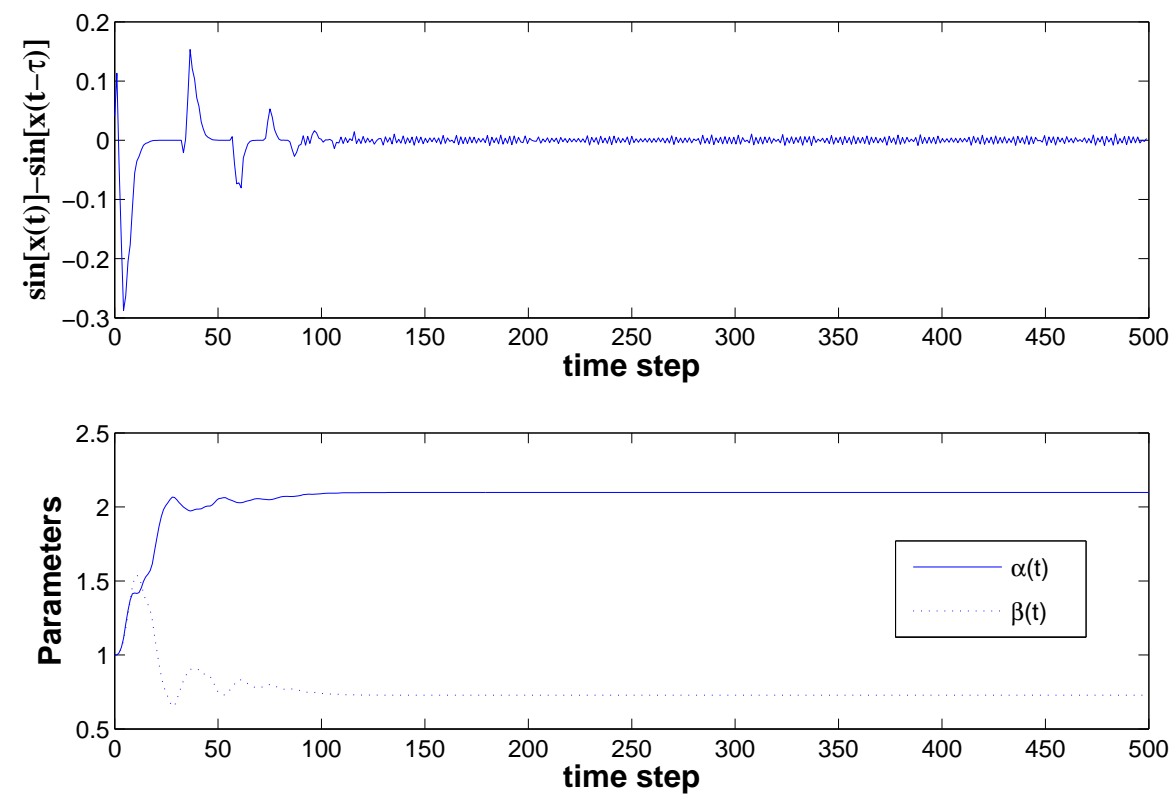

Fig. 8: (a) Successful complete synchronization and parameter identification for chaotic driving signal when $a=-2$ and $b=4$; (b) Failed parameter identification when $a=2$ and $b=1$. This failure is simply due to an approximate dependence between $f(x(t))$ and $g(x(t-\tau))$ in a macro scale. Here, both $f$ and $g$ are taken as sinusoid functions, time-delays are taken as $\tau=10, \delta=2$, and time step size is 0.01 . 